\title{
Educación, pedagogía y didáctica en la escuela: encuentros y desencuentros
}

\author{
Education, pedagogy and didactics in the school: Encounters and disagreements
}

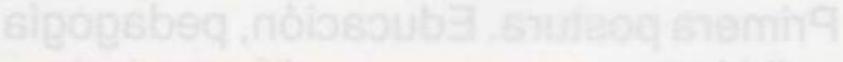
Carmen Alicia Martínez Rivera
Graciela Guzmán Osorio
Alberto Calderón Garcia...

Resumen

Eeste artículo propone aportar una reflexión sobre las posibles relaciones entre los conceptos Educación-pedagogia y didáctica, desde una perspectiva que, sin pretender ser exhaustiva, busca enriquecer posibles miradas y contribuir a que las diferentes disciplinas y saberes especificos, articulen acciones y reflexiones frente a su desarrollo académico.

A partir de dos posturas, ampliamente referenciadas, a que la diferenciación entre pedagogía y didáctica solo se da en algunos aspectos especificos, y su proyección hacia el futuro, es la de ofrecer una perspectiva profesional al docente que trascienda el carácter de tecnicista o transmisor de contenidos.

Sin embargo, este planteamiento requiere continuar el análisis de conceptos centrales en la profesión de maestro. En esa profundización podemos aproximarnos a interrogantes tales como: cuáles son las diferencias entre pedagogía didáctica y educación, que se está asumiendo desde las propuestas educativas? o, cuáles son las concepciones de los maestros?
\end{abstract}

Palabras clave: Didáctica, docentes, educación, pedagogia.

\section{Abstract}

This article proposes we make a reflection about the eventual relations between the concepts of education, pedagogy and didactics based on an unexhaustive perspective. This looks for enriching new approaches and contributing to the expression of actions and reflections through several disciplines and specific knơwledges.

Based on two postures explained widely, it is concluded that the differentiation between pedagogy and didactics is only in some specific aspects, and their future scope offers to the teachers a professional perspective if they go beyond the contents transmitter character.

However, this approach requires the continuation of the central concepts analysis in theteacher profession. In getting inside this situation we can approximate to these questions: Which are the differences between education, pedagogy and didactics? What is it carrying out from the educational proposals? Which are the teachers' concepts?

Key words:

Didactics, teachers, education, pedagogy.

Fecha de recepción: 8 de agosto de 2005 .

Fecha de áceptación: 25 de octubre de 2005.

* Este artículo fue presentado como ponencia en el Encuentro de Facultades de Educación del Suroriente Colombiano. Universidad del Tolima, 2002.

... Profesora de la Facultad de Ciencias y del doctorado en Educación, Universidad del Tolima.

**... Facultad de Educación, Universidad del Tolima.

**** Profesor de la Facultad de Educación Física y del doctorado en Educación, Universidad Pedagógica Nacional. 


\section{Introducción}

Uno de los desafíos que actualmente tenemos los profesores es el reconocimiento y la identificación de nuestro conocimiento como un "conocimiento profesional" que tiene como base el conocimiento disciplinario (quími$\mathrm{ca}$, física, educación física, etc.), el conocimiento pedagógico y didáctico, y la práctica profesional, entre otros aspectos. En este proceso se hace relevante la diferencia de los conceptos que orientan nuestra profesión; en tal sentido, en este escrito nos proponemos aportar una reflexión sobre las posibles relaciones entre los conceptos educación, pedagogía y didáctica, desde una perspectiva que, sin pretender ser exhaustiva, busca enriquecer posibles enfoques.

En un primer apartado presentamos algunas propuestas que establecen diferencias fundamentales entre estos tres saberes; y en el siguiente, algunas tendencias que, a nuestra manera de entender, acercan algunos de estos conceptos entre sí.

Por todos son conocidos los grandes desafíos que tiene hoy la institución educativa. De acuerdo con la Ley General de Educación (ley 115 de 1994), algunos de los fines de la educación apuntan a la solución de nuestros problemas, $y$ a formar ciudadanos críticos $y$ tolerantes. La ley 30 , que rige la educación superior en Colombia, plantea entre sus objetivos contribuir a la formación integral de los colombianos, trabajar por la creación, el desarrollo y la utilización del conocimiento en todas sus formas y expresiones para solucionar las necesidades del país, contribuir al desarrollo de otros niveles educativos, promover la formación y consolidación de comunidades académicas, y promover la preservación del medio ambiente. En la ley 115 se señala que la educación debe favorecer el pleno desarrollo de la personalidad del educando, trabajar en la formación de valores éticos, morales, ciudadanos y religiosos, que le faciliten la realización de una actividad útil para el desarrollo socioeconómico del país. Además, se plantea que el educador es el orientador del proceso de formación de los educandos, acorde con las expectativas socioculturales eficaces y morales de la familia y de la sociedad. Para la formación profesores, el decreto 272 señala:

Los programas académicos en educación tienen el compromiso con la sociedad de formar profesionales capaces de promover acciones formativas, individuales y colectivas, y de comprender y actuar ante la problemática educativa en la perspectiva del desarrollo Integral humano sostenible...
Si pretendemos asumir el compromiso que nuestra profesión demanda hoy, es necesario explicitar y reflexionar acerca de cómo entendemos nuestro quehacer y qué estamos haciendo en relación con él.

\section{Primera postura. Educación, pedagogía y didáctica: tres campos diferenciados}

Teniendo como punto de partida a Rafael Flórez (1991), y con él a Emilio Durkheim, podría afirmarse que la educación hace referencia al conjunto de prácticas propias de un tiempo y un lugar determinados, con las cuales las generaciones adultas entregan a las generaciones jóvenes los resultados de su experiencia. A estos mismos procesos los llama Carlos Vasco (1990) "formación", y emplea el término educación para referirse a la ocurrencia de hechos formativos en contextos institucionales. Según este autor:

La formación se da siempre a partir del nacimiento, $\mathrm{y}$ al menos hasta la edad en la que en cada cultura se considera al joven como ya iniciado en su comunidad. Pero en la terminología propuesta, las prácticas de crianza no institucionalizadas no son propiamente educación, sino formación (p. 9).

Lucio (1989) entiende la educación como el proceso mediante el cual una sociedad, de manera intencional o difusa, promueve el crecimiento de sus individuos. Entendida así la educación, puede ser intencionada o no, institucional o no y reflexionada o no. Lo anterior nos lleva a concluir que, entonces, los procesos educativos son desarrollados por la escuela, también por la familia, por los medios de comunicación, por los amigos, etcétera.

Así mismo, para Lucio la pedagogía es un saber explícito y reflexionado sobre la educación. Al respecto afirma:

Hay pedagogía cuando se reflexiona sobre la educación, cuando el "saber educar" implícito se convierte en un "saber sobre la educación" (sobre sus "cómo", sus "por qué", sus "hacia dónde") (p. 36).

La reflexión sobre los asuntos de la educación, y la manera de concebirla es lo que constituye para Flórez la pedagogía.

De esta manera, educación y pedagogía se diferencian: si el proceso educativo es institucionalizado, explícito y refle-xionado, entonces nos referimos a la pedagogía; en caso contrario, a la educación. En este aspecto coincide Ávila, al señalar que la educación puede ser consciente o inconsciente, mientras que la pedagogía es siempre consciente (Ávila, 1988, citado por Lucio, 1989). 
Vasco (1990) identifica la pedagogía como:

El saber teórico-práctico generado por los pedagogos a través de la reflexión personal y dialogal sobre su propia práctica pedagógica, específicamente en el proceso de convertirla en praxis pedagógica, a partir de su propia experiencia y de los aportes de las otras prácticas y disciplinas que se interceptan con su quehacer (p. 11).

También se diferencia un saber particular en el que se reflexiona sobre la enseñanza: la didáctica (Lucio, 1989), entendiendo la enseñanza como un momento particular de la práctica educativa, y la didáctica como un componente de la pedagogía.

Si la enseñanza es un momento específico, importante aunque no único, del proceso educativo, la didáctica será también un componente importante (aunque tampoco único) de la pedagogía (p. 38).

También para Vasco la didáctica es un sector delimitado de la pedagogía, dedicada a la enseñanza. Este mismo autor señala que su planteamiento es semejante al de Lucio.

De tal manera que las relaciones educación-pedagogía-didáctica se dan en un proceso de menor a mayor focalización, en el cual la educación contempla un proceso más amplio, pues aborda los fenómenos educativos que se dan tanto dentro como fuera de la institución, que incluye la pedagogía, en la cual el fenómeno educativo se hace en una forma intencional, reflexiva y explícita, y esta, a su vez, incluye la didáctica, en la cual la reflexión pedagógica se centra en los procesos de enseñanza. Esta relación puede representarse así:

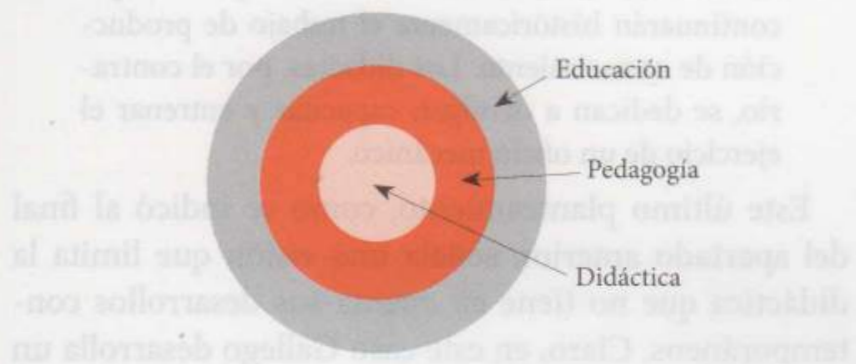

Figura 1. Educación, pedagogía y didáctica, como tres campos de focalización diferente.

Pero si la enseñanza es la institucionalización del quehacer educativo, es de resaltar que en el planteamiento anterior parece que está haciéndose referencia a la enseñanza como algunos la entienden: como un proceso de transmisión de saberes específicos, centrados únicamente en las disciplinas (química, física, etc.) y no, al "quehacer educativo", como la promoción de valores, de actitudes, etc., teniendo en cuenta el contexto, el ambiente emocional, etc. Por ello la diferencia entre pedagogía y didáctica parece que se limita sólo a si el quehacer educativo es institucional o no.

Más aún, esta diferenciación puede contener varios riesgos, puesto que si se señala que "la pedagogía responde científicamente a la pregunta ¿Cómo educar?, la didáctica lo hace a la pregunta ¿Cómo enseñar?” (Lucio, 1989). Podríamos caer en el riesgo de hacer excluyentes la enseñanza y la educación, y entonces podría entenderse la enseñanza centrada en contenidos disciplinarios que en nada contribuyen a la educación, aun cuando la pedagogía como la didáctica "tienen su horizonte específico: el del ¿Cómo educar?”.

La pedagogía es la ciencia que orienta la labor del educador. La didáctica orienta un aspecto específico en ella: su labor como docente... La didáctica se expresa en un currículo (más aún, algunas corrientes alemanas llaman a la didáctica la ciencia del currículo), mientras que la pedagogía lo hace en un programa educativo o en un proyecto pedagógico. La didáctica se concretiza en el aula de clase, que tiene a la escuela como su entorno. La pedagogía escolar puede concretarse en la escuela como grupo humano, que tiene al sistema educativo de la sociedad particular como entorno. La pedagogía familiar en la familia, etc." (Lucio, 1989).

Estas diferencias contribuyen a una visión limitada de la didáctica, de la enseñanza y de la labor docente, que hoy en día es bastante cuestionada. Así por ejemplo, Lucio dice,

Si bien la didáctica puede manejarse como un saber autónomo, con objetivos y metodologías propios, como toda ciencia necesita un horizonte; al perderlo, el saber por el saber se convierte en un fin. Y el horizonte de la didáctica debe ser la pedagogía, así como el horizonte de la pedagogía es una concepción determinada del hombre, de su crecer en sociedad.

Las diferencias entre estos términos pueden corresponderse con los orígenes de los mismos:

Paidogoogía (del griego pais, paidos, niño, niños, y agoo, agein, conduzco, conducir), designa en sus orígenes al esclavo encargado de llevar al niño a la paidea, la escuela. La didascaleia fue la escuela de la instrucción. Didaxis constituyó la lección. Didácalos era el maestro encargado de instruir al infante en la escritura y la lectura... (Gallego, 1990).

Sin embargo, lo que hoy se entiende es algo distinto. Gallego (1990) señala los orígenes del término didáctica en Wolfang Ratke (1571-1635), quien usó, de 1612 a 1618 , el término "didáctico" para identificar al encarga- 
do de estudiar los principios y procedimientos de la enseñanza. Posteriormente, señala Gallego, encontramos a Comenio con la Didáctica magna, en la que se entiende la didáctica como "el arte de enseñar a todos en el menor tiempo posible" (p. 15), según lo planteado en el capítulo XIX, titulado: "Bases para fundar la rapidez de la enseñanza con ahorro de tiempo y fatiga”.

En este sentido, Vasco (1990) también limita la enseñanza cuando dice:

No estoy, pues, de acuerdo con reducir la pedagogía a una reflexión sobre la enseñanza. Quedarían por fuera de esa reflexión muchas relaciones pedagógicamente importantes, como las relaciones de maestros y alumnos con los saberes científicos y con los saberes tradicionales, tanto intraescolares como extra-escolares; las demás relaciones con el macroentorno social y los microentornos sociales y fisicos, en particular las relaciones de los alumnos entre sí y las relaciones entre los maestros..." (p. 17).

Implícitamente está señalándose que la enseñanza no contempla todos estos aspectos, por ello la limita y, por ende, limita la didáctica, aunque más adelante este autor señala:

La didáctica cubre la reflexión sobre todos los aspectos de las relaciones del maestro con sus alumnos y sus microentornos desde un punto de vista de la enseñanza (p. 21), con lo cual amplia un poco más la visión sobre la enseñanza, aunque, aun así, la limita a lo que llama los microentornos, como si la enseñanza no se hiciera preguntas respecto de los macroentornos.

Nuestro planteamiento es que estas diferencias obedecen a una mirada particular sobre la didáctica. Así que en el apartado siguiente mostraremos otra postura que, a nuestro modo de ver, contempla los desarrollos contemporáneos de la didáctica, en particular de la didáctica de las ciencias. Incluso, el mismo Vasco, al reflexionar sobre la pedagogía en la universidad, señala la importancia de que los profesores universitarios reflexionen sobre los procesos de comunicación que allí se dan, $y$ afirma

Es verdad que la pedagogía, o más bien la didáctica como propuesta de métodos de enseñanza, puede convertirse, $y$ de hecho a veces se convierte en una excusa para no seguir aprendiendo e investigando en la propia área en la que se ejerce la docencia, y en un sustituto muy pobre para la falta de conocimientos, de entusiasmo, de espíritu investigativo, y de compromiso con la ciencia respectiva. (p. 25).

Con lo cual, en últimas, invita a reflexionar acerca de cómo entendemos la pedagogía y la didáctica, pues entenderlas como método de enseñanza es una pers- pectiva que aporta poco en la dinámica profesional del profesor universitario.

\section{Segunda postura. Pedagogía y didáctica: dos campos convergentes}

En este apartado señalamos algunos planteamientos en los cuales diferentes autores no establecen diferencia entre la pedagogía y la didáctica, y en la que se enfatiza que ni la pedagogía ni la didáctica pueden entenderse actualmente como procesos técnicos.

Gallego (1990) parte de señalar que:

La pedagogía no es el discurso sobre la educación, ni sobre la enseñanza, ni mucho menos sobre el aprendizaje, actividades estas que la mayoría de las veces son descritas en el discurrir didáctico (p. 19). Este autor hace un planteamiento histórico desde el cual señala que la escuela pitagórica es ejemplo de paideia, en tanto que busca el alcance de la máxima racionalidad de entonces, por conducir todo el proceso bajo su guía y hacia la expresión más elevada de ella, como reguladora de la actividad del pensamiento y de las relaciones entre los hombres, $y$ de los mismos con el cosmos (p. 22).

En estas referencias históricas, este autor destaca el papel que ha cumplido el desarrollo de las ciencias, y la búsqueda de su incorporación en los currículos. En este sentido, diferencia la pedagogía y la didáctica, así:

La ciencia es en su naturaleza y su formulación estrictamente pedagógica, y son los científicos quienes constantemente se preocupan por ella, en el afán de formar los cuadros de investigadores que continuarán históricamente el trabajo de producción de conocimiento. Los didactas, por el contrario, se dedican a divulgar, capacitar y entrenar el ejercicio de un oficio mecánico.

Este último planteamiento, como se indicó al final del apartado anterior, señala una visión que limita la didáctica que no tiene en cuenta sus desarrollos contemporáneos. Claro, en este caso Gallego desarrolla un planteamiento sobre la pedagogía, desde la cual se hace necesaria una "revolución pedagógica":

Requerimos una revolución copernicana, de una transformación mental, de un cambio radical en las interrelaciones que establecemos con nosotros mismos, con el espacio geográfico en el cual habitamos, con la sociedad a la cual pertenecemos y con el saber que ésta posee de sí misma y de su relación con la naturaleza y con otras sociedades de la Tierra. Tenemos que saltar a una concepción diferente del papel de la vida humana, de nuestro rol en este mundo. ¡Y es posible!” (p. 25). 
En este sentido, Gallego define la pedagogía como un proceso de transformación intelectual: En particular, algunas de las transformaciones intelectuales señaladas son:

a. Paso del egocentrismo y el individualismo primitivo, al sociocentrismo colaborador.

b. Del sometimiento a los avatares de la naturaleza animista y considerada consciente, al dominio de la misma, mediante una estructura científica y tecnológica.

c. De la creencia en un orden social dado, en una jerarquía impuesta por potencias sobrenaturales de las cuales es ella representante en la Tierra a la conciencia de que es una estructura creada por los hombres mismos... (pp. 40-41).

Ahora bien, Flórez (1994) hace referencia a la pedagogía como la disciplina que busca la transición hacia una mayoría de edad:

La pedagogía es una disciplina que estudia y propone estrategias para lograr la transición del niño del estado natural al estado humano, hasta la mayoría de edad como ser racional autoconsciente y libre (pp.xxxii).

También se refiere a ella como

...un conjunto coherente de proposiciones que intentan describir y explicar en forma sistemática los procesos educativos relacionados con la enseñanza y el aprendizaje humano (p. xxxviii).

Sin embargo, Flórez, en el análisis epistemológico de la pedagogía, señala que construir una epistemología de la pedagogía tiene una dificultad, relacionada con que muchas veces se señala lo que es pedagogía con otros nombres. En este sentido, invita a reflexionar acerca de los conceptos, las proposiciones, hipótesis, que explican $y$ reconstruyen el proceso de enseñanza-aprendizaje en general, o en un contenido particular, a veces denominada psicopedagogía, o ciencias del currículo, o psicología educativa.

Quizá se trataría más bien de una misma disciplina que bajo otros nombres se propone explicar íntegramente el proceso de enseñanza-aprendizaje, como lo intentan hacer en sus obras investigadores contemporáneos, como Jerónimo Brunner, D. P. Ausubel, K. Lovell, etc. (p. xli). Nos sentimos identificados con este último planteamiento, sólo que le adicionaríamos la didáctica. Pues la poca claridad conceptual que se vive entorno de la pedagogía, también se da en la didáctica. Así, autores como Parlebas (1992) señalan esta divergencia respecto a la conceptualización sobre didáctica:

Se pueden contar tantas definiciones de didáctica, como autores que han escrito sobre ella. El único punto en el que todos están de acuerdo es en que no han podido llegar a un consenso acerca del significado preciso del término. Incluso, señala Parlebas, pueden encontrarse posturas en las que la didáctica es asumida como una ciencia en especial o general que contendría a la pedagogía:

Actualmente está dándose entre didáctica y pe-
dagogía una especie de inversión de significado.
Mientras que la primera se consideraba general-
mente una disciplina que hacía parte de la pedago-
gía, hoy existe una manifiesta tendencia a invertir
el esquema. De esta forma, la didáctica alcanzaría
el nivel de ciencia, especial o general; de teoría que
contendría a la pedagogía y, por tanto, se conver-
tiría en ciencia o en metodología de la enseñanza
y de la apropiación de conocimientos. Algunos,
más osados aún, se refieren a ella como estudio
del funcionamiento del sistema de transmisión de
los saberes disciplinarios.

También Noguera, mencionando por Sáenz-López (1997), enfatiza la diversidad de maneras de entender la didáctica:

a. Es ciencia.

b. Es arte.

c. Es el estudio de los procesos de enseñanza-aprendizaje.

d. Supone una constante toma de decisiones normativas.

e. Es teórico-práctica.

A continuación se mostrará cómo los planteamientos actuales sobre la pedagogía se encuentran también en la didáctica contemporánea, por lo menos en los escritos sobre la didáctica de las ciencias.

Flórez (1994) señala a la pedagogía como una disciplina científica en construcción: "Asumimos provisionalmente que la pedagogía contemporánea es una disciplina científica dispersa aún bajo diferentes denominaciones y teorías..." (p. xliii).

Plantea que educar es desarrollar la capacidad creadora, la capacidad de asimilar y producir cultura; entonces, la pedagogía. Es la disciplina que desarrolla y sistematiza el saber acerca del cómo de la educación, en el contexto cultural de una forma social particular (p. 21). La pedagogía busca el desarrollo intelectual de los niños, busca remover los obstáculos para el aprendizaje de cada ciencia, como el subjetivismo, el animismo, el antropomorfismo, la causalidad única, etc. $\mathrm{Al}$ respecto, es de destacar que estos obstáculos del aprendizaje son retomados por Flórez del libro de Aebli titulado: Didáctica: una aplicación de la psicología de Jean Piaget. Lo anterior ejemplifica la denominación indiferenciada que se ha hecho entre pedagogía y 
didáctica: se alude a la pedagogía, pero se retoma de lo referido a la didáctica.

Flórez (1994) dice que el pedagogo define los contenidos, las experiencias, el lenguaje y las metodologías adecuados para la enseñanza de los nuevos conocimientos y para el desarrollo interpersonal y social que contribuye al desarrollo de la personalidad y la creatividad del alumno (pp.88). Reitera que educar es mucho más que enseñar ciencias:

La prevención contra una educación centrada en la enseñanza de las ciencias se justificaría sólo como concesión a la concepción positivista reinante en el siglo XX sobre la enseñanza de las ciencias (como mecanismo para reproducirse como comunidad científica); concepción traída a nuestro medio sin recibir críticas, y propuesta recientemente como objeto reducido de la pedagogia, que desarraiga la enseñanza de la vida de los alumnos y de las necesidades reales de aplicación y transformación (p. 95).

Estamos de acuerdo en que si se entiende la enseñanza como un proceso positivista centrado en el aprendizajes de contenidos acabados, entonces sería necesario diferenciar la didáctica de la pedagogía. Flórez señala más adelante que la enseñanza de las ciencias debe contribuir a la formación integral del ser humano, por ello no puede centrarse ni en contenidos acabados, ni en métodos de enseñanza. Se entiende la formación como el proceso mediante el cual se alcanza la mayoría de edad: Formar... es facilitarle que asuma en su vida su propia dirección racional, reconociendo fraternalmente a sus semejantes el mismo derecho y la misma dignidad (p. 111).

Hasta aquí hemos presentado lo que entendemos por pedagogía como disciplina y cuál es su objeto de estudio. Veamos ahora su relación con las visiones contemporáneas sobre la didáctica de las ciencias.

En principió, es de destacar que la didáctica de las ciencias es entendida como una disciplina o como una disciplina emergente (Martínez, 2000), en tanto que se encuentra en proceso de consolidación de las características que la hacen posible. Porlán (1998), retomando a Toulmin (1972), indica las condiciones que debe cumplir un cuerpo de conocimiento para ser considerado una disciplina:

a. Un conjunto de problemas específicos, conceptuales o prácticos.

b. La existencia de una comunidad profesional crítica.

c. Un punto de vista general y compartido sobre la disciplina (metas e ideales).

d. Estrategias y procedimientos aceptados.

e. Poblaciones conceptuales en evolución vinculadas a los problemas específicos (p. 19).
De esta manera, las preguntas que en su momento se han formulado respecto de la pedagogía como ciencia (Aebli, 1994), también podemos plantearlas hoy respecto de la didáctica: a) ¿Cuál es la función de la didáctica? b) ¿Cuál es el cuerpo de conocimiento que constituye a la didáctica? c) ¿Qué metodología de investigación se usa en la didáctica.? d) ¿Cuál es la relación teórico-práctica en la didáctica?

La didáctica de las ciencias deja de ser entendida como un conjunto de técnicas. Hoy se señala la necesidad de un conocimiento específico que aborde los problemas específicos de enseñanza-aprendizaje de las ciencias (Gil et al, 2000).

La didáctica tiene como finalidad analizar y ofrecer alternativas sobre los problemas de los procesos de enseñanza-aprendizaje:

La finalidad de la enseñanza de las ciencias. Presenta dos dimensiones complementarias: describir y analizar los problemas más significativos de la enseñanza-aprendizaje de las ciencias y elaborar y experimentar modelos que, a la luz de los problemas detectados, ofrezcan alternativas prácticas fundamentadas y coherentes (Porlán, 1996).

De tal manera que se asume como objeto de estudio de la didáctica de las ciencias el sistemas de enseñanza y aprendizaje.

Podría decirse que asistimos a un momento fructífero del conocimiento en la didáctica, que se nota, por ejemplo, en las diferentes publicaciones que sobre la didáctica de las ciencias vienen realizándose, y cuyo número se ha incrementado a partir de la década de 1980 (Gil y otros, 2000). Al respecto, Porlán (1998) propone cuatro problemáticas para el desarrollo de la didáctica:

a. Profundizar en los fines y fundamentos de un modelo alternativo de enseñanza-aprendizaje de las ciencias (...) La teoría sistémica, la teoría de la complejidad, la teoría evolucionista y la teoría crítica son, entre otras, aportaciones relevantes. (...)

b. Desarrollar una nueva teoría del conocimiento escolar y de las estrategias que favorecen su construcción. (...)

c. Desarrollar una nueva teoría del conocimiento profesional y de las estrategias que favorecen su construcción. (...)

d. Diseñar y experimentar propuestas de formación del profesorado que, tomando como referencia los avances en los puntos anteriores, tengan como orientación fundamental promover y apoyar, a su vez, la experimentación de hipótesis curriculares superadoras de los problemas que plantea el modelo tradicional de enseñanza aprendizaje de las ciencias." (p.36). 
Cabe destacar que, entendidas así, tanto la pedagogía como la didáctica, éstas son asumidas como disciplinas en construcción y que, contemporáneamente, buscan atender a los problemas de la enseñanza desde una perspectiva amplia; esto es, no centrada en contenidos acabados, sino que pretende la formación integral. De acuerdo con Campos y Restrepo (1999):

\begin{abstract}
Una educación encaminada a la formación integral asume al ser humano como persona íntegra, como totalidad, es una educación que reconoce las dimensiones humanas en constante interrelación, no por partes, sino en permanente despliegue, presentes siempre en todos los ámbitos de la existencia; es una educación que reconoce que en cualquier actividad educativa está implicada la persona como un todo; en consecuencia, ni privilegia la inteligencia sobre la afectividad, ni separa la imaginación de la acción, ni dicotomiza el desarrollo individual del social.
\end{abstract}

Todo lo anterior, porque se ha concebido la formación como un proceso permanente de construcción del ser de la persona.

Desde la perspectiva de una búsqueda de la formación integral, la didáctica comprende un espacio para la reflexión y el cambio socioprofesional, contextualizado en un determinado marco histórico y político, desde una opción constructivista, tanto del conocimiento humano como de los procesos de enseñanza y aprendizaje (Grupo Investigación en la Escuela, 1991). Por ello, el modelo didáctico es entendido como "un estructurador que organiza, describe y explica distintas opciones/realidades educativas" (p. 6).

De este modo, Flórez $(1994,1999)$ identifica los modelos pedagógico que se diferencian, desde el punto de vista de los contenidos, así:

\section{El tradicional}

centrado en disciplinas y autores clásicos, resultados de la ciencia.

\section{El conductista}

centrado en conocimientos técnicos, códigos, destrezas y competencias observables.

\section{El romanticismo pedagógico}

sin ninguna programación, sólo la que el alumno solicite.

\section{El desarrollismo pedagógico:}

Contenidos centrados en experiencias que faciliten acceso a estructuras superiores de desarrollo, el niño construye sus propios contenidos de aprendizaje.

\section{La pedagogía socialista}

Con contenidos científico-técnico, polifacético y politécnico.

Según el grupo de investigación DIE (didáctica e Investigación Escolar) (Martínez, 2000), desde la didáctica de las ciencias se identifican los siguientes modelos didácticos:

\section{Modelo tradicional}

Centrado en los contenidos. Los contenidos escolares se asumen como "reproducción simplificada del conocimiento disciplinario", por tanto, son de carácter terminal y prefijado.

\section{Modelo tecnológico}

Centrado en la definición exhaustiva de objetivos. Aquí los contenidos son "adaptaciones del conocimiento disciplinario", adopta y se una visión cientificista y experimentalista del proceso de enseñanza y aprendizaje.

\section{Modelo espontaneista}

Centrado en el alumno. El contenido se asume como "adaptación contextual del conocimiento cotidiano", centrado en los intereses de los estudiantes.

\section{Modelo alternativo}

Resalta el carácter complejo y participativo del proceso de enseñanza y aprendizaje; en este modelo el contenido es la "integración de conocimientos que proceden de diversas fuentes" (ideas de los alumnos, problemáticas relevantes del contexto sociocultural, conocimiento científico, conocimientos metadisciplinares).

En esta propuesta se destaca que el conocimiento escolar genera sus propios procesos de construcción, diferentes de los que se dan en las instituciones científicas, $\mathrm{y}$ de los que se dan en la sociedad en general (Martínez, 2000).

Si comparamos la clasificación de modelos pedagógicos y modelos didácticos, expuesta antes, solamente en función de los contenidos, vemos que en ambos casos se plantea una clasificación que va desde modelos en los cuales los contenidos son acabados (son los productos de las ciencias), hasta modelos más complejos en los cuales el conocimiento científico es uno más de los contenidos, y en los cuales se da una perspectiva más compleja. Como dice Flórez (1994) al describir el modelo de pedagogía socialista: El desarrollo intelectual no se identifica con el aprendizaje, como creen los conductistas, ni se produce independientemente del aprendizaje de la ciencia, como creen los desarrollistas (p. 170). 
Porlán (1998) señala que se ha venido dando un cambio en el propósito de enseñar, se ha pasado de una enseñanza centrada en la ciencia a una enseñanza que busca contribuir a la formación científica de todos los ciudadanos (Martínez, 2000), con lo cual se da una cercanía en las dos tendencias, a pesar de que la primera se refiere a modelos pedagógicos y la segunda a modelos didácticos.

Por lo anterior, no es posible continuar haciendo referencia a la didáctica como un conjunto de técnicas, sino reconocer que se trata de una disciplina que está en proceso de construcción (proceso en el que también se encuentra la pedagogía). pedagogía y didáctica se han venido acercando cada vez más y, por ello, de acuerdo con esta postura, podría representarse a la didáctica y a la pedagogía (figura 2) como dos disciplinas que tienden a indiferenciarse, en tanto que:

a. Ambas pretenden una formación integral, no centrada en contenidos acabados

b. Para ambas el conocimiento científico es importante, mas no es el fin en sí mismo

c. Ambas pretenden la formación de ciudadanos críticos, autónomos y creativos

d. Ambas buscan desarrollar una postura crítica frente a los procesos tradicionales

e. Ambas se asumen como conocimientos reflexionados y explícitos de los profesores que investigan y analizan su práctica.

f. Ambas pretenden hacer explícitos y criticables los fundamentos ideológicos de cualquier sistema educativo.

\section{Bibliografía}

ÁVILA PENAGOS, Rafael (1991). Pedagogía y autorregulación cultural. Colección Pedagogía Siglo XXI. Bogotá. Ediciones Antropos.

CAMPOS V., Rafael y RESTREPO J., Mariluz (1999). Formación integral. Facultad de Educación. Santa Fe de Bogotá. Pontificia Universidad Javeriana.

FLÓREZ OCHOA, Rafael (1999). Evaluación pedagogía y cognición. Bogotá. McGraw-Hill.

. (1994). Hacia una pedagogía del conocimiento. Bogotá.

McGraw-Hill.

GALLEGO BADILLO, R. (1990). Saber pedagógico. Bogotá. Magisterio.

GIL, D. y otros (2000). Una disciplina emergente y un campo específico de investigación. En Perales y Cañal (Comps). La didáctica de las ciencias experimentales. Alcoy. Marfil.

LUCIO, R. (1989). Educación y pedagogía, enseñanza y didáctica: diferencias y relaciones. Bogotá. Revista de la Universidad de la Salle. Número 17.

MARTINEZ RIVERA, C. (2000). Las propuestas curriculares de los profesores sobre el conocimiento escolar: dos estudios de caso

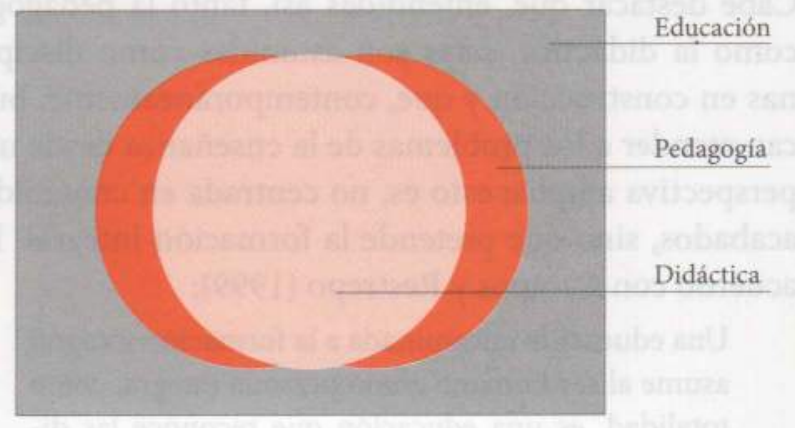

Figura 2. Pedagogia y didáctica, disciplinas en convergencia.

A manera de conclusión, podríamos afirmar que en la primera postura la diferencia entre pedagogía y didáctica es tajante y que todavía se encuentra bastante arraigada en diferentes contextos; sin embargo, la segunda postura, en la cual la diferenciación entre pedagogía y didáctica solo se da en algunos aspectos específicos, es la que se abre paso en estos momentos, y se proyecta hacia el futuro, al ofrecer una perspectiva profesional del docente que trasciende el carácter tecnicista o de transmisión de contenidos. Pero este planteamiento requiere una mayor profundización. Así, cabe preguntarse: ¿Cuáles son las diferencias que está asumiendo desde las propuestas educativas? ¿Cuáles son las concepciones de los maestros?. Retomamos nuevamente el planeamiento inicial, pues en el proceso de diferenciación del conocimiento profesional de los profesores es necesario continuar en un enfoque que permita analizar conceptos centrales de nuestra profesión.

en el área del conocimiento del medio. Tesis doctoral. Universidad de Sevilla, España.

MINISTERIO DE EDUCACIÓN NACIONAL. (1998). Criterios $y$ procedimientos para la acreditación previa de los programas académicos de pregrado y de especialización en educación.

(1994). Ley 115 de febrero 8.

PARLEBAS, Pierre (1992-1993). Didáctica y lógica de las actividades físicas deportivas. Educación Física y Deporte. Vols. 14-15, enero-diciembre. Medellín. Universidad de Antioquía. Instituto Universitario de Educación Física y Deporte.

PORLÁN, R. (1998). Pasado, presente y futuro de la didáctica de las Ciencias. Enseñanza de las Ciencias, 16(1).

SÁENZ-LÓPEZ BUÑUEL, Pedro (1997). La didáctica de la educación física. En La educación física y su didáctica. Manual para el profesor. España. Wanceulen Editorial Deportiva, SL.

VASCO, C. E. (1990). Reflexiones sobre pedagogía y didáctica. Bogotá. Ministerio de Educación Nacional.

(1988). Algunas reflexiones entorno de la pedagogía. En Revista Actualidad en Educación. 